\title{
Efficiency of NNBD over NNBIBD using First Order Correlated Models
}

\author{
R. Senthil kumar ${ }^{1}$ and C. Santharam ${ }^{2}$ \\ ${ }^{1,2}$ Department of Statistics, Loyola College, Chennai- 600034 (TN), INDIA.
}

\begin{abstract}
Neighbour Balanced Block Designs, permitting the estimation of direct and neighbour effects, are used when the treatment applied to one experimental plot may affect the response on neighbouring plots as well as the response on the plot to which it is applied. The allocation of treatments in these designs is such that every treatment occurs equally often with every other treatment as neighbours. Neighbour Balanced Block Designs for observations correlated within a block have been investigated for the estimation of direct as well as left and right neighbour effects of treatments. It is observed that efficiency for direct as well as neighbour effects is high, in case of Complete block designs i.e., $(m=0)$ for Nearest Neighbour correlation structure with $\rho$ in the interval 0.1 to 0.4. In case of incomplete block designs $(m=1,2, \ldots, v-4)$ for Nearest Neighbour correlation structure turns out to be more efficient as compared to others models with $\rho$ in the interval 0.1 to 0.4. The performance of Nearest neighbour balanced block designs is satisfactory for ARMA(1,1) models. The gain in efficiency of NNBD and NNBIBD over regular block design is high under MA(1) models for direct and neighbour effects of treatments.
\end{abstract}

Keywords: Neighbour Balanced Block Design; Correlated observations; Generalized least squares; AutoRegressive; Moving Average; Nearest neighbour; Efficiency; Regular Block Design.

\section{Introduction:}

Experiments conducted in agriculture often show neighbour effects i.e., the response on a given plot is affected by the treatments on the neighbouring plots as well as by the treatment applied to that particular plot. When treatments are varieties, neighbour effects may be caused by differences in height, root vigor, or germination date especially on small plots, which are used in plant breeding experiments. Treatments such as fertilizer, irrigation, or pesticide may spread to adjacent plots causing neighbour effects. Such experiments exhibit neighbour effects, because the effect of having no treatment as a neighbour is different from the neighbour effects of any treatment. Competition or interference between neighbouring units in field experiments can contribute to variability in experimental results and lead to substantial losses in efficiency. In case of block design setup, if each block is a single line of plots and blocks are well separated, extra parameters are needed for the effect of left and right neighbours. An alternative is to have border plots on both ends of every block. Each border plot receives an experimental treatment, but it is not used for measuring the response variable. These border plots do not add too much to the cost of one-dimensional experiments.

Neighbour balanced block designs, where in the allocation of treatments is such that every treatment occurs equally often with every other treatment as neighbours, are used for modeling and controlling interference effects between neighbouring plots. Azais et al. (1993) obtained a series of efficient neighbour designs with border plots that are balanced in $v-1$ blocks of size $v$ and $v$ blocks of size $v-1$, where $v$ is the number of treatments. In the analysis of data from comparative experiments, the correlation structure among observation is often unknown. If due care is taken in allocating the treatments by randomization, then the usual analysis assuming independence of observations is approximately valid, Kempthorne (1952). However, in situations where the correlation structure is known it may be advantageous to use this information in designing an experiment and analyzing the data so as to make more precise inference, Williams (1952); Bartlett (1978); Kiefer and Wynn (1981); Martin (1982); Wilkinson et al. (1983). In field experiments block of small sizes are effective in removing heterogeneity due to variation in fertility. In some cases blocks may represent additive effects not necessarily related to local fertility variation. The effect of correlation on the usual two-way analysis of variance and on the power of usual tests has been studied by Box (1954); Anderson, Jenson and Schou (1981) and Aastveit (1983). Highly efficient neighbour balanced designs are introduced by Kunert (1987) for positive correlated structure.

The construction of nearest neighbour balanced designs with partial variance balance was introduced by Morgan and Chakravarti, (1988). Optimal and highly efficient two dimensional designs were constructed for correlated errors on the torus and in the plane by Morgen and Nizamuddin (1991). Druilhet (1999) studied optimality of circular neighbour balanced block designs obtained by Azais et al. (1993). Bailey (2003) has given some designs for studying one-sided neighbour effects. These neighbour balanced block designs have been 
developed under the assumption that the observations within a block are uncorrelated. In situations where the correlation structure among the observations within a block is known, may be from the data of past similar experiments, it may be advantageous to use this information in designing an experiment and analyzing the data so as to make more precise inference about treatment effects Gill and Shukla (1985). Kunert et al. (2003) considered two related models for interference and have shown that optimal designs for one model can be obtained from optimal designs for the other model. Martin and Eccelston (2004) have given variance balanced designs under interference and dependent observations. Tomar and Seema Jaggi (2007) observed that efficiency is quite high, in case of complete block designs for both AR(1) and NN correlation structures. Santharam.C \& K.N.Ponnuswamy (1997) observed that the performance of NNBD is quite satisfactory for the remaining models. In this paper, neighbour balanced block designs for observations correlated within a block have been investigated for the estimation of direct as well as left and right neighbour effects of treatments. The performance of these designs for $\operatorname{AR}(1), \operatorname{MA}(1)$ and $\operatorname{ARMA}(1,1)$ and $\mathrm{NN}$ error correlation structure is studied when generalized least squares estimation is used. We have also investigated the efficiency of Nearest Neighbour Balanced Block Design (NNBD) and Nearest Neighbour Balanced Incomplete Block Design (NNBIBD) in comparison to regular block design when the error follows first order correlated models.

\section{Model Structures And Information Matrix:}

Let $\Delta$ be a class of binary neighbour balanced block designs with $n=b k$ units that form b blocks each containing k units. $Y_{i j}$ be the response from the $i^{\text {th }}$ plot in the $j^{\text {th }}$ block $(i=1,2, \ldots, k ; j=1,2, \ldots, b)$. The layout includes border plots at both ends of every block, i.e. at $0^{\text {th }}$ and $(k+1)^{\text {th }}$ position and observations for these units are not modeled. The following fixed effects additive model is considered for analyzing a neighbour balanced block design under correlated observations:

$$
Y_{i j}=\mu+\tau_{(i, j)}+l_{(i-1, j)}+\gamma_{(i+1, j)}+\beta_{j}+e_{i j}
$$

where $\mu$ is the general mean, $\tau_{(i, j)}$ is the direct effect of the treatment in the $i^{\text {th }}$ plot of $j^{\text {th }}$ block, $\beta_{j}$ is the effect of the $j^{\text {th }}$ block. $l_{(i-1, j)}$ is the left neighbour effect due to the treatment in the $(i-1)^{\text {th }}$ plot of $j^{\text {th }}$ block. $\gamma_{(i+1, j)}$ is the right neighbour effect due to the treatment in the $(i+1)^{\text {th }}$ plot in $j^{\text {th }}$ block. $e_{i j}$ are error terms distributed with mean zero and a variance-covariance structure $\Omega=I_{b} \otimes \Lambda$ ( $I_{b}$ is an identity matrix of order $b$ and $\otimes$ denotes the kronecker product). Assuming no correlation among the observations between the blocks and correlation structure between plots within a block to be the same in each block, $\Lambda$ is the correlation matrix of $k$ observations within a block. The error-in variable model Besag (1977) is closely related to the smooth trend plus error model of Wilkinson et al. (1983). This is a general model which gives a better fit in situations where the error structure is non stationary Besag (1977); Wilkinson et al. (1983) Patterson (1983). Gill and Shukla (1985) studied universal optimality of NNBD using AR(1) and MA(1) models. The $\operatorname{ARMA}(1,1)$ model along with $\mathrm{AR}(1)$ and $\mathrm{MA}(1)$ and explored the performance of NNBD for $\rho=-0.4(-0.4) 0.4$. If the errors within a block follow a $\mathbf{A R ( 1 ) ~ s t r u c t u r e , ~ t h e n ~} \Lambda$ is a $k \times k$ matrix with $\left(i, i^{\prime}\right)^{t h}$ entry $\left(i, i^{\prime}=1,2, \ldots, k\right)$ as $\rho^{\left|i-i^{\prime}\right|},|\rho|<1$. The MA(1) structure, then $\Lambda$ is a matrix with diagonal entries as 1 and $\left(i, i^{\prime}\right)^{t h}$ entry $\left(i, i^{\prime}=1,2, \ldots, k\right)$ as $\rho$, when $\left|i-i^{\prime}\right|=1$, otherwise zero Gill and Shukla, (1985). If the errors within a block follow an $\operatorname{ARMA(1,1)~model~then~} \Omega=I_{b} \otimes \Lambda$. Where $I_{b}$ is an identity matrix of order $b$ and $\Lambda=\left[\begin{array}{ccccc}r_{0} & r_{1} & r_{2} & \ldots & r_{k-1} \\ r_{1} & r_{0} & r_{1} & \ldots & r_{k-2} \\ r_{2} & r_{1} & r_{0} & \ldots & r_{k-3} \\ & & & \vdots & \\ r_{k-1} & r_{k-2} & r_{k-3} & \ldots & r_{0}\end{array}\right]$, where $\quad r_{0}=\frac{1+2 \rho_{1} \rho_{2}+\rho_{2}^{2}}{1-\rho_{1}^{2}}$, 
$r_{1}=\frac{\rho_{1}\left(1+\rho_{2}^{2}\right)+\rho_{2}\left(1+\rho_{1}^{2}\right)}{1-\rho_{1}^{2}}, r_{k}=\rho_{1}^{r}(k-1)$ for $k \geq 2$, Santharam.C \& K.N.Ponnuswamy (1997). The NN correlation structure, the $\Lambda$ is a matrix with diagonal entries as 1 and off-diagonal entries as $\rho$.

Model (2.1) can be rewritten in the matrix notation as follows:

$$
Y=\mu 1+\Delta^{\prime} \tau+\Delta_{1}^{\prime} l+\Delta_{2}^{\prime} \gamma+D^{\prime} \beta+e
$$

where $Y$ is $n \times 1$ vector of observations, 1 is $n \times 1$ vector of ones, $\Delta^{\prime}$ is an $n \times v$ incidence matrix of observations versus direct treatments, $\tau$ is $v \times 1$ vector of direct treatment effects, $\Delta_{1}^{\prime}$ is a $n \times v$ matrix of observations versus left neighbour treatment, $\Delta_{2}^{\prime}$ is a $n \times v$ matrix of observations versus right neighbour treatment, $l$ is $v \times 1$ vector of left neighbour effects, $\gamma$ is $v \times 1$ vector of right neighbour effects, $D^{\prime}$ is an $n \times b$ incidence matrix of observations versus blocks, $\beta$ is $b \times 1$ vector of block effects and $e$ is $n \times 1$ vector of errors. The joint information matrix for estimating the direct and neighbour (left and right) effects under correlated observations estimated by generalized least squares is obtained as follows:

$$
C=\left[\begin{array}{ccc}
\Delta\left(I_{b} \otimes \wedge^{*}\right) \Delta^{\prime} & \Delta\left(I_{b} \otimes \wedge^{*}\right) \Delta_{1}^{\prime} & \Delta\left(I_{b} \otimes \wedge^{*}\right) \Delta_{2}^{\prime} \\
\Delta_{1}\left(I_{b} \otimes \wedge^{*}\right) \Delta^{\prime} & \Delta_{1}\left(I_{b} \otimes \wedge^{*}\right) \Delta_{1}^{\prime} & \Delta_{1}\left(I_{b} \otimes \wedge^{*}\right) \Delta_{2}^{\prime} \\
\Delta_{2}\left(I_{b} \otimes \wedge^{*}\right) \Delta^{\prime} & \Delta_{2}\left(I_{b} \otimes \wedge^{*}\right) \Delta_{1}^{\prime} & \Delta_{2}\left(I_{b} \otimes \wedge^{*}\right) \Delta_{2}^{\prime}
\end{array}\right]
$$

with

$$
\wedge^{*}=\wedge^{-1}-\left(1_{k}^{\prime} \wedge^{-1} 1_{k}\right)^{-1} \wedge^{-1} 1_{k} 1_{k}^{\prime} \wedge^{-1}
$$

The above $3 v \times 3 v$ information matrix $(C)$ for estimating the direct effects and neighbour effects of treatments in a block design setting is symmetric, non-negative definite with row and column sums equal to zero. The information matrix for estimating the direct effects of treatments from (2.3) is as follows:

$$
C_{\tau}=C_{11}-C_{12} C_{22}^{-1} C_{21}
$$

where

$$
\begin{aligned}
& C_{11}=\Delta\left(I_{b} \otimes \wedge^{*}\right) \Delta^{\prime} \\
& C_{12}=\left[\Delta\left(I_{b} \otimes \wedge^{*}\right) \Delta_{1}^{\prime} \quad \Delta\left(I_{b} \otimes \wedge^{*}\right) \Delta_{2}^{\prime}\right] \quad \text { and } \\
& C_{22}=\left[\begin{array}{ll}
\Delta_{1}\left(I_{b} \otimes \wedge^{*}\right) \Delta_{1}^{\prime} & \Delta_{1}\left(I_{b} \otimes \wedge^{*}\right) \Delta_{2}^{\prime} \\
\Delta_{2}\left(I_{b} \otimes \wedge^{*}\right) \Delta_{1}^{\prime} & \Delta_{2}\left(I_{b} \otimes \wedge^{*}\right) \Delta_{2}^{\prime}
\end{array}\right]
\end{aligned}
$$

Similarly, the information matrix for estimating the left neighbour effect of treatments $\left(C_{l}\right)$ and right neighbour effect of treatments $\left(C_{\gamma}\right)$ can be obtained.

\subsection{Construction Of Design:}

Tomer et al. (2005) has constructed neighbour balanced block design with parameters $v$ (prime or prime power $), b=v(v-1), r=(v-1)(v-m), k=(v-m), m=1,2, \ldots, v-4$ and $\lambda=(v-m)$ using Mutually Orthogonal Latin Squares (MOLS) of order $v$. This series of design bas been investigated under the correlated error structure. It is seen that the design turns out to be pair-wise uniform with $\alpha=1$ and also variance balanced for estimating direct $\left(V_{1}\right)$ and neighbour effects $\left(V_{2}=V_{3}\right)$.

\section{Comparison Of Efficiency:}

In this section, a quantitative measure of efficiency of the designs in compared to the universally optimal neighbour balanced design for $v$ treatments in $(v-1)$ complete blocks of Azais et al. (1993) considering observations to be correlated within the blocks. We compare the average variance of an elementary treatment contrast $\hat{\tau}_{s}-\hat{\tau}_{s^{\prime}}$ in both cases. The average variance of an elementary treatment contrast 
Kempthorne (1956) for direct effects of the neighbour balanced design of Azais et al. (1993) estimated by generalized least squares methods, is given by

$$
V_{A}=\frac{2 \sigma^{2}}{v-1} \sum_{s=1}^{v-1} \theta_{s}^{-1}
$$

Where $\theta_{s}$ 's are the $(v-1)$ non-zero eigen values of $C_{\tau}$ for Azais et al. (1993), $\sigma^{2}$ is the variance of an observation. The efficiency factor $\left(E_{\tau}\right)$ for direct effects of the neighbour balanced pair-wise uniform block design is thus given as:

$$
E_{\tau}=\frac{(v-1) \sum_{s=1}^{v-1} \theta_{s}^{-1}}{(v-m) \sum_{s=1}^{v-1} \delta_{s}^{-1}}
$$

$\delta_{s}$ 's are the $(v-1)$ non-zero eigen values of $C_{\tau}$. Similarly the efficiency $\left(E_{l}\right)$ and $\left(E_{\gamma}\right)$ for neighbour effects (left and right) of treatments is obtained. The ranges of correlation coefficient $(\rho)$ for different correlation structures investigated are $|\rho| \leq 0.40$ for $\mathrm{AR}(1), \mathrm{MA}(1), \mathrm{ARMA}(1,1)$ and $\mathrm{NN}$ correlation structures. For these ranges, the matrix of correlation coefficients among observations within a block is positive definite. For $\rho=0$, the efficiency is that of totally balanced designs obtained by Tomer et al. (2005). These matrices have been worked out using $\mathbf{R}$ package.

In Tables 1, 2, $3 \& 4$, the parameters of neighbour balanced pair-wise uniform block design for $v=5$ $(m=0,1)$ and $v=6(m=0,1,2)$ along with the efficiency for direct and neighbour effects (left and right) has been shown. The efficiency values have been reported under the $\operatorname{AR}(1), \operatorname{MA}(1), \operatorname{ARMA}(1,1)$ and NN correlation structures with $\rho$ in the interval -0.4 to 0.4. It is seen that efficiency for direct as well as neighbour effects is high, in case of Complete block designs i.e., $(m=0)$ for NN correlation structure with $\rho$ in the interval 0.1 to 0.4 . In case of incomplete block designs $(m=1,2, \ldots, v-4)$ for NN correlation structure turns out to be more efficient as compared to others models with $\rho$ in the interval 0.1 to 0.4 . The performance of Nearest neighbour balanced block designs is satisfactory for ARMA(1,1) models. Moreover, as the block size increases, the efficiency also increases because the plots in a block become more heterogeneous. So we concluded that when block sizes are large and neighbouring plots are highly correlated, generalized least squares for estimation of direct and neighbour effects can be used.

\subsection{Efficiency of NNBD and NNBIBD in comparison to Regular Block Design (RBD):}

For a regular block design the variance of an elementary treatment contrast, estimated by ordinary least squares methods, is given by

$$
V_{1}=2 r^{-1} \sigma^{2}(1-\bar{\rho})
$$

where $\sigma^{2}$ is the variance of an observation, $\bar{\rho}$ is the average correlation between observations from any two plots within a block, the average being taken over all possible randomizations; $r$ is the number of replications. If observations within a block follow an errors-in-variable autoregressive model, then Williams (1952)

and

$$
\sigma^{2}=\sigma_{\varepsilon}^{2}\left(1+\alpha\left(1-\rho^{2}\right)\left(1-\rho^{2}\right)^{-1}\right)
$$

$$
\bar{\rho}=\frac{2 \rho}{\left\{1+\alpha\left(1-\rho^{2}\right)(t-1)(1-\rho)\right.}\left\{1-\frac{1-\rho^{t}}{t(1-\rho)}\right\}
$$

so that $\quad V_{1}=\frac{2 \sigma_{\varepsilon}^{2}}{r\left(1-\rho^{2}\right)}\left[1+\alpha\left(1-\rho^{2}\right)-\frac{2}{(t-1)(1-\rho)}\left\{1-\frac{\left(1-\rho^{t}\right)}{t(1-\rho)}\right\}\right]$

When errors follow a first order moving average model, then

When errors follow first order ARMA model, then

$$
V_{1}=2 r^{-1} \sigma_{\varepsilon}^{2}\left(1+\rho^{2}\right)\left(1+\frac{\alpha}{1+\rho^{2}}-\frac{2}{\left(1+\rho^{2}\right) t}\right)
$$




$$
V_{1}=2 r^{-1} \sigma_{\varepsilon}^{2} r_{0}\left(1+\frac{\alpha}{r_{0}}-\frac{2}{t(t-1)} \sum_{s=1}^{(t-1)}(t-s) \gamma_{s}\right)
$$

where $\alpha=\frac{\sigma_{\eta}^{2}}{\sigma_{\varepsilon}^{2}}$

For generalized least squares estimation, the average variance of an elementary treatment contrast, estimated from a design $d$, is

$$
V_{2}=\frac{2 \sigma^{2}}{v-1} \sum_{s=1}^{v-1} \theta_{s}^{-1}
$$

where $\theta_{s}$ 's are non-zero values of $C_{d}$ (Kempthorne, 1956).

We define the efficiency of a design $d$ relative to a regular block design as $V_{1} / V_{2}$.

The Tables 5 and 6 shows the efficiencies of NNBD with $t=5, r=20$ and $t=6, r=30, \rho=-0.4$ to 0.4 and $\alpha=1$. The Tables 7 and 8 shows the efficiencies of NNBIBD with $t=5, r=16$ and $t=6, r=25$, $\rho=-0.4$ to 0.4 and $\alpha=1$. The values in the tables show that as $\rho$ increases from 0.1 to 0.4 the gain in efficiency also increases under $\operatorname{AR}(1), \operatorname{MA}(1)$ and $\operatorname{ARMA}(1,1)$ models. The gain in efficiency of NNBD and NNBIBD over regular block design is high under MA(1) models for direct and neighbour effects of treatments.

\section{Tables:}

\begin{tabular}{|c|c|c|c|c|c|c|c|c|}
\hline \multirow{2}{*}{\multicolumn{5}{|c|}{ Parameters }} & \multirow{2}{*}{\multicolumn{4}{|c|}{$\begin{array}{c}\text { Correlation Structure } \\
\mathbf{A R}(1) \\
\end{array}$}} \\
\hline & & & & & & & & \\
\hline$v$ & $b$ & $m$ & $r$ & $k=\lambda$ & $\rho$ & $E_{\tau}$ & $E_{l}$ & $E_{\gamma}$ \\
\hline \multirow[t]{9}{*}{5} & 20 & 0 & 20 & 5 & -0.4 & 0.68287 & 0.63750 & 0.64130 \\
\hline & & & & & -0.3 & 0.70822 & 0.69768 & 0.71440 \\
\hline & & & & & -0.2 & 0.77781 & 0.75809 & 0.73164 \\
\hline & & & & & -0.1 & 0.77054 & 0.76368 & 0.76484 \\
\hline & & & & & 0.0 & 0.80000 & 0.80000 & 0.80000 \\
\hline & & & & & 0.1 & 0.82721 & 0.82792 & 0.82486 \\
\hline & & & & & 0.2 & 0.88525 & 0.86802 & 0.86477 \\
\hline & & & & & 0.3 & 0.94868 & 0.95093 & 0.96874 \\
\hline & & & & & 0.4 & 1.02361 & 0.98896 & 0.94436 \\
\hline \multirow[t]{9}{*}{5} & 20 & 1 & 16 & 4 & -0.4 & 0.91927 & 0.77656 & 0.78475 \\
\hline & & & & & -0.3 & 0.90071 & 0.89407 & 0.84008 \\
\hline & & & & & -0.2 & 0.92510 & 0.87165 & 0.87467 \\
\hline & & & & & -0.1 & 0.91861 & 0.95754 & 0.95331 \\
\hline & & & & & 0 & 1.00000 & 1.00000 & 1.00000 \\
\hline & & & & & 0.1 & 1.06857 & 1.02668 & 1.06492 \\
\hline & & & & & 0.2 & 1.15298 & 1.13899 & 1.13881 \\
\hline & & & & & 0.3 & 1.26678 & 1.27000 & 1.26607 \\
\hline & & & & & 0.4 & 1.43687 & 1.36980 & 1.37313 \\
\hline \multirow[t]{9}{*}{6} & 30 & 0 & 30 & 6 & -0.4 & 0.75486 & 0.77745 & 0.70181 \\
\hline & & & & & -0.3 & 0.76514 & 0.53448 & 0.76891 \\
\hline & & & & & -0.2 & 0.77722 & 0.78902 & 0.79626 \\
\hline & & & & & -0.1 & 0.79679 & 0.80511 & 0.80286 \\
\hline & & & & & 0.0 & 0.80000 & 0.80000 & 0.80000 \\
\hline & & & & & 0.1 & 0.86984 & 0.86448 & 0.85899 \\
\hline & & & & & 0.2 & 0.90461 & 0.80481 & 0.90171 \\
\hline & & & & & 0.3 & 0.95909 & 0.93856 & 0.94975 \\
\hline & & & & & 0.4 & 1.03151 & 0.99695 & 1.01706 \\
\hline \multirow[t]{6}{*}{6} & 30 & 1 & 25 & 5 & -0.4 & 0.94070 & 0.90147 & 0.92474 \\
\hline & & & & & -0.3 & 0.94336 & 0.93130 & 0.97118 \\
\hline & & & & & -0.2 & 0.95051 & 0.94902 & 0.92849 \\
\hline & & & & & -0.1 & 0.96449 & 0.92726 & 0.99197 \\
\hline & & & & & 0 & 1.00000 & 1.00000 & 1.00000 \\
\hline & & & & & 0.1 & 1.04849 & 1.05117 & 1.05435 \\
\hline
\end{tabular}

Table 1. Efficiency of neighbour balanced pair-wise uniform block designs AR(1) model: 


\begin{tabular}{|c|c|c|c|c|c|c|c|c|}
\hline \multirow{10}{*}{6} & \multirow{10}{*}{30} & \multirow{10}{*}{2} & \multirow{10}{*}{20} & \multirow{10}{*}{4} & $\begin{array}{l}0.2 \\
0.3 \\
0.4\end{array}$ & $\begin{array}{l}1.12566 \\
1.22454 \\
1.36957 \\
\end{array}$ & $\begin{array}{l}1.12695 \\
1.20896 \\
1.34255 \\
\end{array}$ & $\begin{array}{l}1.11943 \\
1.22333 \\
1.34608 \\
\end{array}$ \\
\hline & & & & & -0.4 & 1.28188 & 1.24096 & 1.26246 \\
\hline & & & & & -0.3 & 1.24104 & 1.27744 & 1.27200 \\
\hline & & & & & -0.2 & 1.14425 & 1.27770 & 1.27733 \\
\hline & & & & & -0.1 & 1.25492 & 1.29384 & 1.28132 \\
\hline & & & & & 0 & 1.25000 & 1.25000 & 1.25000 \\
\hline & & & & & 0.1 & 1.13327 & 1.32053 & 1.35493 \\
\hline & & & & & 0.2 & 1.40332 & 1.42642 & 1.42774 \\
\hline & & & & & 0.3 & 1.50318 & 1.51162 & 1.39422 \\
\hline & & & & & 0.4 & 1.50767 & 1.63851 & 1.66997 \\
\hline
\end{tabular}

Table 2. Efficiency of neighbour balanced pair-wise uniform block designs MA(1) model:

\begin{tabular}{|c|c|c|c|c|c|c|c|c|}
\hline & & Par & ters & & & Corre & Structur & \\
\hline & & & & & & & (1) & \\
\hline$v$ & $b$ & $m$ & $r$ & $k=\lambda$ & $\rho$ & $E_{\tau}$ & $E_{l}$ & $E_{\gamma}$ \\
\hline 5 & 20 & 0 & 20 & 5 & -0.4 & 0.66631 & 0.63644 & 0.66063 \\
\hline & & & & & -0.3 & 0.71832 & 0.69436 & 0.69760 \\
\hline & & & & & -0.2 & 0.76633 & 0.70641 & 0.71013 \\
\hline & & & & & -0.1 & 0.76103 & 0.77614 & 0.74742 \\
\hline & & & & & 0.0 & 0.80000 & 0.80000 & 0.80000 \\
\hline & & & & & 0.1 & 0.81629 & 0.84870 & 0.83885 \\
\hline & & & & & 0.2 & 0.86839 & 0.86158 & 0.86363 \\
\hline & & & & & 0.3 & 0.90889 & 0.91996 & 0.88717 \\
\hline & & & & & 0.4 & 0.93365 & 0.88157 & 0.88499 \\
\hline 5 & 20 & 1 & 16 & 4 & -0.4 & 0.83067 & 0.79043 & 0.82266 \\
\hline & & & & & -0.3 & 0.85458 & 0.89807 & 0.87499 \\
\hline & & & & & -0.2 & 0.91680 & 0.90371 & 0.90170 \\
\hline & & & & & -0.1 & 0.94057 & 0.91259 & 0.94392 \\
\hline & & & & & 0 & 1.00000 & 1.00000 & 1.00000 \\
\hline & & & & & 0.1 & 1.01894 & 1.08297 & 1.08979 \\
\hline & & & & & 0.2 & 1.13295 & 1.15707 & 1.12392 \\
\hline & & & & & 0.3 & 1.23253 & 1.17668 & 1.18523 \\
\hline & & & & & 0.4 & 1.35526 & 1.26243 & 1.26446 \\
\hline 6 & 30 & 0 & 30 & 6 & -0.4 & 0.72261 & 0.69922 & 0.71010 \\
\hline & & & & & -0.3 & 0.73073 & 0.73851 & 0.75539 \\
\hline & & & & & -0.2 & 0.77151 & 0.78147 & 0.77456 \\
\hline & & & & & -0.1 & 0.79159 & 0.80102 & 0.81227 \\
\hline & & & & & 0.0 & 0.80000 & 0.80000 & 0.80000 \\
\hline & & & & & 0.1 & 0.88099 & 0.85001 & 0.86476 \\
\hline & & & & & 0.2 & 0.89240 & 0.89178 & 0.88415 \\
\hline & & & & & 0.3 & 0.92133 & 0.91276 & 0.90509 \\
\hline & & & & & 0.4 & 0.94970 & 0.87412 & 0.92125 \\
\hline 6 & 30 & 1 & 25 & 5 & -0.4 & 0.89115 & 0.86763 & 0.90908 \\
\hline & & & & & -0.3 & 0.91019 & 0.91106 & 0.97813 \\
\hline & & & & & -0.2 & 0.93529 & 0.93476 & 0.93494 \\
\hline & & & & & -0.1 & 0.97401 & 0.97891 & 0.96524 \\
\hline & & & & & 0 & 1.00000 & 1.00000 & 1.00000 \\
\hline & & & & & 0.1 & 1.05547 & 1.06322 & 1.05580 \\
\hline & & & & & 0.2 & 1.11470 & 1.10227 & 1.12250 \\
\hline & & & & & 0.3 & 1.18969 & 1.17620 & 1.18041 \\
\hline & & & & & 0.4 & 1.29669 & 1.24530 & 1.27229 \\
\hline 6 & 30 & 2 & 20 & 4 & -0.4 & 1.16993 & 1.19977 & 1.20830 \\
\hline & & & & & -0.3 & 1.19463 & 1.22385 & 1.25078 \\
\hline & & & & & -0.2 & 1.21384 & 1.26023 & 1.25781 \\
\hline
\end{tabular}




\begin{tabular}{|l|c|c|c|c|} 
& -0.1 & 1.26544 & 1.30056 & 1.31168 \\
& 0 & 1.25000 & 1.25000 & 1.25000 \\
& 0.1 & 1.32778 & 1.31478 & 1.33994 \\
& 0.2 & 1.38415 & 1.39339 & 1.35076 \\
& 0.3 & 1.46225 & 1.23450 & 1.39011 \\
& 0.4 & 1.54402 & 1.27473 & 1.40892 \\
\hline
\end{tabular}

Table 3. Efficiency of neighbour balanced pair-wise uniform block designs ARMA(1,1) model:

\begin{tabular}{|c|c|c|c|c|c|c|c|c|}
\hline \multirow{2}{*}{\multicolumn{5}{|c|}{ Parameters }} & \multirow{2}{*}{\multicolumn{4}{|c|}{ Correlation Structure }} \\
\hline & & & & & & & & \\
\hline$v$ & $b$ & $m$ & $r$ & $k=\lambda$ & $\rho$ & $E_{\tau}$ & $E_{l}$ & $E_{\gamma}$ \\
\hline \multirow[t]{9}{*}{5} & 20 & 0 & 20 & 5 & $(-0.4,-0.4)$ & 0.32275 & 0.41998 & 0.34200 \\
\hline & & & & & $(-0.3,-0.3)$ & 0.44297 & 0.25651 & 0.27193 \\
\hline & & & & & $(-0.2,-0.2)$ & 0.58318 & 0.58497 & 0.55448 \\
\hline & & & & & $(-0.1,-0.1)$ & 0.69314 & 0.70919 & 0.71026 \\
\hline & & & & & $(0,0)$ & 0.80000 & 0.80000 & 0.80000 \\
\hline & & & & & $(0.1,0.1)$ & 0.83073 & 0.82819 & 0.82177 \\
\hline & & & & & $(0.2,0.2)$ & 0.78885 & 0.73788 & 0.75456 \\
\hline & & & & & $(0.3,0.3)$ & 0.63023 & 0.34204 & 0.18718 \\
\hline & & & & & $(0.4,0.4)$ & 0.58709 & 0.06418 & 0.30422 \\
\hline \multirow[t]{9}{*}{5} & 20 & 1 & 16 & 4 & $(-0.4,-0.4)$ & 0.41672 & 0.26870 & 0.40328 \\
\hline & & & & & $(-0.3,-0.3)$ & 0.59656 & 0.49940 & 0.54387 \\
\hline & & & & & $(-0.2,-0.2)$ & 0.63500 & 0.74506 & 0.71194 \\
\hline & & & & & $(-0.1,-0.1)$ & 0.89426 & 0.86752 & 0.85693 \\
\hline & & & & & $(0,0)$ & 1.00000 & 1.00000 & 1.00000 \\
\hline & & & & & $(0.1,0.1)$ & 1.04927 & 1.09501 & 1.07778 \\
\hline & & & & & $(0.2,0.2)$ & 1.10968 & 1.03478 & 1.05581 \\
\hline & & & & & $(0.3,0.3)$ & 0.66680 & 0.57787 & 0.87287 \\
\hline & & & & & $(0.4,0.4)$ & 0.94687 & 0.81728 & 0.60362 \\
\hline \multirow[t]{9}{*}{6} & 30 & 0 & 30 & 6 & $(-0.4,-0.4)$ & 0.36038 & 0.42080 & 0.62225 \\
\hline & & & & & $(-0.3,-0.3)$ & 0.49118 & 0.36038 & 0.45027 \\
\hline & & & & & $(-0.2,-0.2)$ & 0.62365 & 0.60793 & 0.61215 \\
\hline & & & & & $(-0.1,-0.1)$ & 0.83020 & 0.74801 & 0.74261 \\
\hline & & & & & $(0,0)$ & 0.80000 & 0.80000 & 0.80000 \\
\hline & & & & & $(0.1,0.1)$ & 0.86001 & 0.84611 & 0.84876 \\
\hline & & & & & $(0.2,0.2)$ & 0.80037 & 0.75366 & 0.75237 \\
\hline & & & & & $(0.3,0.3)$ & 0.65451 & 0.70426 & 0.60153 \\
\hline & & & & & $(0.4,0.4)$ & 0.56920 & 0.70866 & 0.43601 \\
\hline \multirow[t]{9}{*}{6} & 30 & 1 & 25 & 5 & $(-0.4,-0.4)$ & 0.41672 & 0.26870 & 0.40328 \\
\hline & & & & & $(-0.3,-0.3)$ & 0.59656 & 0.49940 & 0.54387 \\
\hline & & & & & $(-0.2,-0.2)$ & 0.63500 & 0.74506 & 0.71194 \\
\hline & & & & & $(-0.1,-0.1)$ & 0.89426 & 0.86752 & 0.85693 \\
\hline & & & & & $(0,0)$ & 1.00000 & 1.00000 & 1.00000 \\
\hline & & & & & $(0.1,0.1)$ & 1.04927 & 1.09501 & 1.07778 \\
\hline & & & & & $(0.2,0.2)$ & 1.10968 & 1.03478 & 1.05581 \\
\hline & & & & & $(0.3,0.3)$ & 0.66680 & 0.57787 & 0.87287 \\
\hline & & & & & $(0.4,0.4)$ & 0.94687 & 0.81728 & 0.60362 \\
\hline \multirow[t]{9}{*}{6} & 30 & 2 & 20 & 4 & $(-0.4,-0.4)$ & 0.60324 & 0.55304 & 0.59972 \\
\hline & & & & & $(-0.3,-0.3)$ & 0.80020 & 0.80436 & 0.80408 \\
\hline & & & & & $(-0.2,-0.2)$ & 1.00430 & 1.06373 & 1.02336 \\
\hline & & & & & $(-0.1,-0.1)$ & 1.16018 & 1.20070 & 1.13169 \\
\hline & & & & & $(0,0)$ & 1.25000 & 1.25000 & 1.25000 \\
\hline & & & & & $(0.1,0.1)$ & 1.32576 & 1.28337 & 1.28064 \\
\hline & & & & & $(0.2,0.2)$ & 1.27443 & 1.07083 & 1.18289 \\
\hline & & & & & $(0.3,0.3)$ & 1.17668 & 1.15822 & 0.81134 \\
\hline & & & & & $(0.4,0.4)$ & 0.95160 & 1.40105 & 1.47460 \\
\hline
\end{tabular}


Table 4. Efficiency of neighbour balanced pair-wise uniform block designs NN model:

\begin{tabular}{|c|c|c|c|c|c|c|c|c|}
\hline \multirow{2}{*}{\multicolumn{5}{|c|}{ Parameters }} & \multicolumn{4}{|c|}{ Correlation Structure } \\
\hline & & & & & & & & \\
\hline$v$ & $b$ & $m$ & $r$ & $k=\lambda$ & $\rho$ & $E_{\tau}$ & $E_{l}$ & $E_{\gamma}$ \\
\hline \multirow[t]{9}{*}{5} & 20 & 0 & 20 & 5 & -0.4 & 0.56287 & 0.53710 & 0.56352 \\
\hline & & & & & -0.3 & 0.62530 & 0.60242 & 0.58364 \\
\hline & & & & & -0.2 & 0.66174 & 0.65598 & 0.66174 \\
\hline & & & & & -0.1 & 0.71680 & 0.71779 & 0.72109 \\
\hline & & & & & 0.0 & 0.80000 & 0.80000 & 0.80000 \\
\hline & & & & & 0.1 & 0.89193 & 0.94829 & 0.90732 \\
\hline & & & & & 0.2 & 0.99359 & 0.99120 & 1.02672 \\
\hline & & & & & 0.3 & 1.13873 & 1.17027 & 1.15689 \\
\hline & & & & & 0.4 & 1.37226 & 1.36867 & 1.33303 \\
\hline \multirow[t]{9}{*}{5} & 20 & 1 & 16 & 4 & -0.4 & 0.71506 & 0.69636 & 0.69396 \\
\hline & & & & & -0.3 & 0.75236 & 0.74652 & 0.75591 \\
\hline & & & & & -0.2 & 0.82142 & 0.84158 & 0.82263 \\
\hline & & & & & -0.1 & 0.91183 & 0.91525 & 0.89459 \\
\hline & & & & & 0 & 1.00000 & 1.00000 & 1.00000 \\
\hline & & & & & 0.1 & 1.08703 & 1.12496 & 1.12064 \\
\hline & & & & & 0.2 & 1.26785 & 1.26785 & 1.22112 \\
\hline & & & & & 0.3 & 1.46534 & 1.45527 & 1.46279 \\
\hline & & & & & 0.4 & 1.71428 & 1.71428 & 1.71428 \\
\hline \multirow[t]{9}{*}{6} & 30 & 0 & 30 & 6 & -0.4 & 0.58753 & 0.58692 & 0.58983 \\
\hline & & & & & -0.3 & 0.63785 & 0.64068 & 0.62985 \\
\hline & & & & & -0.2 & 0.68384 & 0.69532 & 0.65391 \\
\hline & & & & & -0.1 & 0.75234 & 0.76089 & 0.77723 \\
\hline & & & & & 0.0 & 0.80000 & 0.80000 & 0.80000 \\
\hline & & & & & 0.1 & 0.93761 & 0.91002 & 0.93524 \\
\hline & & & & & 0.2 & 1.05008 & 1.04844 & 1.04667 \\
\hline & & & & & 0.3 & 1.20284 & 1.20351 & 1.20192 \\
\hline & & & & & 0.4 & 1.40943 & 1.40854 & 1.40744 \\
\hline \multirow[t]{9}{*}{6} & 30 & 1 & 25 & 5 & -0.4 & 0.71225 & 0.71208 & 0.71123 \\
\hline & & & & & -0.3 & 0.77117 & 0.76562 & 0.77063 \\
\hline & & & & & -0.2 & 0.83426 & 0.82881 & 0.83152 \\
\hline & & & & & -0.1 & 0.91387 & 0.91031 & 0.81473 \\
\hline & & & & & 0 & 1.00000 & 1.00000 & 1.00000 \\
\hline & & & & & 0.1 & 1.12770 & 1.12770 & 1.11300 \\
\hline & & & & & 0.2 & 1.27373 & 1.27373 & 1.27373 \\
\hline & & & & & 0.3 & 1.45613 & 1.46291 & 1.21291 \\
\hline & & & & & 0.4 & 1.71922 & 1.72950 & 1.50969 \\
\hline \multirow[t]{9}{*}{6} & 30 & 2 & 20 & 4 & -0.4 & 0.89534 & 0.90183 & 0.88105 \\
\hline & & & & & -0.3 & 0.96847 & 0.99492 & 0.98984 \\
\hline & & & & & -0.2 & 1.07332 & 1.12483 & 1.06958 \\
\hline & & & & & -0.1 & 1.15528 & 1.20506 & 1.19129 \\
\hline & & & & & 0 & 1.25000 & 1.25000 & 1.25000 \\
\hline & & & & & 0.1 & 1.39981 & 1.39783 & 1.47445 \\
\hline & & & & & 0.2 & 1.61141 & 1.73902 & 1.64013 \\
\hline & & & & & 0.3 & 1.86870 & 1.92505 & 1.93073 \\
\hline & & & & & 0.4 & 2.14480 & 2.26269 & 2.26082 \\
\hline
\end{tabular}


Table 5. Efficiency of NNBD in comparison to Regular Block Designs:

$$
t=5, r=20 \& \alpha=1
$$

\begin{tabular}{|c|c|c|c|c|c|c|c|c|c|c|}
\hline $\begin{array}{l}\text { ERROR } \\
\text { MODEL }\end{array}$ & & $\rho=-0.4$ & $\rho=-0.3$ & $\rho=-0.2$ & $\rho=-0.1$ & $\rho=0$ & $\rho=0.1$ & $\rho=0.2$ & $\rho=0.3$ & $\rho=0.4$ \\
\hline \multirow[t]{3}{*}{$\operatorname{AR}(1)$} & $E_{z}$ & 1.47814 & 1.46146 & 1.54845 & 1.49238 & 1.51678 & 1.54253 & 1.62946 & 1.72863 & 1.85063 \\
\hline & $E_{l}$ & 1.37991 & 1.43972 & 1.50920 & 1.47910 & 1.51678 & 1.54385 & 1.59775 & 1.73273 & 1.78799 \\
\hline & $E_{\gamma}$ & 1.38815 & 1.47422 & 1.45654 & 1.48135 & 1.51678 & 1.53814 & 1.59176 & 1.76518 & 1.76736 \\
\hline \multirow[t]{3}{*}{$\mathrm{MA}(1)$} & $E_{\tau}$ & 1.38965 & 1.43854 & 1.48927 & 1.45192 & 1.51678 & 1.55734 & 1.68762 & 1.82016 & 1.94719 \\
\hline & $E_{l}$ & 1.32734 & 1.39055 & 1.37283 & 1.48074 & 1.51678 & 1.61918 & 1.67438 & 1.84234 & 1.83859 \\
\hline & $E_{\gamma}$ & 1.37779 & 1.39703 & 1.38005 & 1.42594 & 1.51678 & 1.60039 & 1.67836 & 1.77666 & 1.84571 \\
\hline \multirow[t]{4}{*}{$\operatorname{ARMA}(1,1)$} & & $\begin{array}{l}\rho_{1}=-0.4 \\
\rho_{2}=-0.4\end{array}$ & $\begin{array}{l}\rho_{1}=-0.3 \\
\rho_{2}=-0.3\end{array}$ & $\begin{array}{l}\rho_{1}=-0.2 \\
\rho_{2}=-0.2\end{array}$ & $\begin{array}{l}\rho_{1}=-0.1 \\
\rho_{2}=-0.1\end{array}$ & $\begin{array}{l}\rho_{1}=0 \\
\rho_{2}=0\end{array}$ & $\begin{array}{l}\rho_{1}=0.1 \\
\rho_{2}=0.1\end{array}$ & $\begin{array}{l}\rho_{1}=0.2 \\
\rho_{2}=0.2\end{array}$ & $\begin{array}{l}\rho_{1}=0.3 \\
\rho_{2}=0.3\end{array}$ & $\begin{array}{l}\rho_{1}=0.4 \\
\rho_{2}=0.4\end{array}$ \\
\hline & $E_{z}$ & 0.98867 & 1.20145 & 1.44753 & 1.62780 & 1.84604 & 1.96140 & 1.97832 & 1.73609 & 1.86451 \\
\hline & $E_{l}$ & 1.32456 & 0.97660 & 1.45076 & 1.66676 & 1.84604 & 1.95521 & 1.84707 & 1.92859 & 1.72272 \\
\hline & $E_{\gamma}$ & 1.04873 & 0.91748 & 1.37301 & 1.66932 & 1.84604 & 1.94001 & 1.88997 & 1.49353 & 1.94887 \\
\hline
\end{tabular}

Table 6. Efficiency of NNBD in comparison to Regular Block Designs:

\begin{tabular}{|c|c|c|c|c|c|c|c|c|c|c|}
\hline $\begin{array}{l}\text { ERROR } \\
\text { MODEL }\end{array}$ & & $\rho=-0.4$ & $\rho=-0.3$ & $\rho=-0.2$ & $\rho=-0.1$ & $\rho=0$ & $\rho=0.1$ & $\rho=0.2$ & $\rho=0.3$ & $\rho=0.4$ \\
\hline \multirow[t]{3}{*}{$\mathrm{AR}(1)$} & $E_{\tau}$ & 1.25504 & 1.26007 & 1.26649 & 1.56936 & 1.61061 & 1.65862 & 1.70921 & 1.80218 & 1.93826 \\
\hline & $E_{l}$ & 1.21662 & 1.1182 & 1.59451 & 1.58574 & 1.61061 & 1.64840 & 1.52066 & 1.76360 & 1.87332 \\
\hline & $E_{\gamma}$ & 1.53873 & 1.60865 & 1.60915 & 1.58132 & 1.61061 & 1.63793 & 1.70374 & 1.78462 & 1.91110 \\
\hline \multirow[t]{3}{*}{$\operatorname{MA}(1)$} & $E_{\tau}$ & 1.24456 & 1.29938 & 1.35342 & 1.46817 & 1.61061 & 1.82305 & 2.07825 & 2.45032 & 2.98558 \\
\hline & $E_{l}$ & 1.24326 & 1.30514 & 1.37614 & 1.47943 & 1.61061 & 1.76939 & 2.07500 & 2.45168 & 2.98370 \\
\hline & $E_{\gamma}$ & 1.24944 & 1.28308 & 1.35356 & 1.51121 & 1.61061 & 1.81843 & 2.07151 & 2.44845 & 2.98136 \\
\hline \multirow[t]{5}{*}{$\operatorname{ARMA}(1,1)$} & & $\rho_{1}=-0.4$ & $\rho_{1}=-0.3$ & $\rho_{1}=-0.2$ & $\rho_{1}=-0.1$ & $\rho_{1}=0$ & $\rho_{1}=0.1$ & $\rho_{1}=0.2$ & $\rho_{1}=0.3$ & $\rho_{1}=0.4$ \\
\hline & & $\rho_{2}=-0.4$ & $\rho_{2}=-0.3$ & $\rho_{2}=-0.2$ & $\rho_{2}=-0.1$ & $\rho_{2}=0$ & $\rho_{2}=0.1$ & $\rho_{2}=0.2$ & $\rho_{2}=0.3$ & $\rho_{2}=0.4$ \\
\hline & $E_{\tau}$ & 1.12047 & 1.32899 & 1.53475 & 1.93334 & 1.89949 & 2.00399 & 2.12174 & 1.78502 & 2.05476 \\
\hline & $E_{l}$ & 1.30707 & 0.96501 & 1.49379 & 1.73824 & 1.89949 & 1.97088 & 1.86175 & 1.92420 & 2.23690 \\
\hline & $E_{\gamma}$ & 1.93195 & 1.21224 & 1.50540 & 1.72580 & 1.89949 & 1.97732 & 1.85941 & 1.63881 & 1.36079 \\
\hline
\end{tabular}


Table 7. Efficiency of NNBIBD in comparison to Regular Block Designs:

$$
t=5, r=16 \& \alpha=1
$$

\begin{tabular}{|c|c|c|c|c|c|c|c|c|c|c|}
\hline $\begin{array}{l}\text { ERROR } \\
\text { MODEL }\end{array}$ & & $\rho=-0.4$ & $\rho=-0.3$ & $\rho=-0.2$ & $\rho=-0.1$ & $\rho=0$ & $\rho=0.1$ & $\rho=0.2$ & $\rho=0.3$ & $\rho=0.4$ \\
\hline \multirow[t]{3}{*}{$A R(1)$} & $E_{\tau}$ & 2.40406 & 2.24291 & 1.94392 & 1.78779 & 1.75000 & 1.63277 & 1.84430 & 1.92974 & 2.09111 \\
\hline & $E_{l}$ & 1.60599 & 2.14795 & 2.16361 & 1.67788 & 1.75000 & 1.34688 & 1.80453 & 1.95517 & 2.45095 \\
\hline & $E_{y}$ & 1.68570 & 1.06782 & 1.55923 & 1.45009 & 1.75000 & 1.96296 & 2.05801 & 2.54910 & 2.76561 \\
\hline \multirow[t]{3}{*}{$\mathrm{MA}(1)$} & $E_{\tau}$ & 0.66485 & 1.18572 & 1.40207 & 1.56814 & 1.75000 & 1.89563 & 2.05108 & 2.70763 & 2.88273 \\
\hline & $E_{l}$ & 0.61608 & 0.98563 & 1.25378 & 1.06007 & 1.75000 & 1.99853 & 2.24473 & 2.46932 & 3.04925 \\
\hline & $E_{\gamma}$ & 0.40613 & 0.61851 & 1.06635 & 1.27072 & 1.75000 & 2.23253 & 2.66118 & 3.93841 & 4.99749 \\
\hline \multirow[t]{4}{*}{$\operatorname{ARMA}(1,1)$} & & $\begin{array}{l}\rho_{1}=-0.4 \\
\rho_{2}=-0.4\end{array}$ & $\begin{array}{l}\rho_{1}=-0.3 \\
\rho_{2}=-0.3\end{array}$ & $\begin{array}{l}\rho_{1}=-0.2 \\
\rho_{2}=-0.2\end{array}$ & $\begin{array}{l}\rho_{1}=-0.1 \\
\rho_{2}=-0.1\end{array}$ & $\begin{array}{l}\rho_{1}=0 \\
\rho_{2}=0\end{array}$ & $\begin{array}{l}\rho_{1}=0.1 \\
\rho_{2}=0\end{array}$ & $\begin{array}{l}\rho_{1}=0.2 \\
\rho_{2}=0.2\end{array}$ & $\begin{array}{l}\rho_{1}=0.3 \\
\rho_{2}=0.3\end{array}$ & $\begin{array}{l}\rho_{1}=0.4 \\
\rho_{2}=0.4\end{array}$ \\
\hline & $E_{\tau}$ & 1.02213 & 1.21753 & 1.31032 & 1.00917 & 0.93750 & 0.77900 & 0.99777 & 1.20131 & 1.91861 \\
\hline & $E_{l}$ & 0.33385 & 0.67311 & 0.73347 & 0.89404 & 0.93750 & 0.88992 & 1.12436 & 1.68039 & 1.92382 \\
\hline & $E_{\gamma}$ & 0.36534 & 0.42791 & 0.69098 & 0.83773 & 0.93750 & 1.20479 & 1.70126 & 2.60086 & 2.70172 \\
\hline
\end{tabular}

Table 8. Efficiency of NNBIBD in comparison to Regular Block Designs:

\begin{tabular}{|c|c|c|c|c|c|c|c|c|c|c|}
\hline $\begin{array}{l}\text { ERROR } \\
\text { MODEL }\end{array}$ & & $\rho=-0.4$ & $\rho=-0.3$ & $\rho=-0.2$ & $\rho=-0.1$ & $\rho=0$ & $\rho=0.1$ & $\rho=0.2$ & $\rho=0.3$ & $\rho=0.4$ \\
\hline \multirow[t]{3}{*}{$\mathrm{AR}(1)$} & $E_{\tau}$ & 2.47063 & 2.00443 & 1.77158 & 1.55059 & 1.45713 & 1.45461 & 1.43150 & 1.46008 & 1.70143 \\
\hline & $E_{l}$ & 1.59125 & 1.39874 & 1.43204 & 1.64688 & 1.45713 & 1.53177 & 1.77508 & 1.96795 & 2.25002 \\
\hline & $E_{\gamma}$ & 1.22794 & 1.81426 & 1.71675 & 1.28532 & 1.45713 & 1.57240 & 1.84319 & 2.00079 & 2.39380 \\
\hline \multirow[t]{3}{*}{$\operatorname{MA}(1)$} & $E_{\tau}$ & 0.73380 & 0.93913 & 1.15138 & 1.38203 & 1.45713 & 1.59347 & 1.63743 & 1.90847 & 2.34649 \\
\hline & $E_{l}$ & 0.39902 & 0.67674 & 0.95515 & 1.14646 & 1.45713 & 1.87677 & 2.07172 & 2.62201 & 3.48893 \\
\hline & $E_{\gamma}$ & 0.50268 & 0.71305 & 0.94363 & 1.18529 & 1.45713 & 1.76199 & 2.25254 & 3.03607 & 4.76615 \\
\hline \multirow[t]{4}{*}{$\operatorname{ARMA}(1,1)$} & & $\begin{array}{l}\rho_{1}=-0.4 \\
\rho_{2}=-0.4\end{array}$ & $\begin{array}{l}\rho_{1}=-0.3 \\
\rho_{2}=-0.3\end{array}$ & $\begin{array}{l}\rho_{1}=-0.2 \\
\rho_{2}=-0.2\end{array}$ & $\begin{array}{l}\rho_{1}=-0.1 \\
\rho_{2}=-0.1\end{array}$ & $\begin{array}{l}\rho_{1}=0 \\
\rho_{2}=0\end{array}$ & $\begin{array}{l}\rho_{1}=0.1 \\
\rho_{2}=0.1\end{array}$ & $\begin{array}{l}\rho_{1}=0.2 \\
\rho_{2}=0.2\end{array}$ & $\begin{array}{l}\rho_{1}=0.3 \\
\rho_{2}=0.3\end{array}$ & $\begin{array}{l}\rho_{1}=0.4 \\
\rho_{2}=0.4\end{array}$ \\
\hline & $E_{\tau}$ & 1.23894 & 1.08918 & 0.94535 & 0.84534 & 0.76832 & 0.75204 & 0.76445 & 0.90912 & 1.89671 \\
\hline & $E_{l}$ & 0.52647 & 0.55659 & 0.62387 & 0.65892 & 0.76823 & 0.94086 & 1.07837 & 2.97753 & 3.00972 \\
\hline & $E_{\gamma}$ & 0.52408 & 0.45172 & 0.67446 & 0.64942 & 0.76823 & 0.95559 & 1.43839 & 3.00571 & 3.11432 \\
\hline
\end{tabular}




\section{Conclusion:}

We have concluded that the efficiency for direct as well as neighbour effects is high, in case of Complete and Incomplete block designs for NN correlation structure with $\rho$ in the interval 0.1 to 0.4 . The performance of Nearest neighbour balanced block designs is satisfactory for ARMA $(1,1)$ models. Moreover, as the block size increases, the efficiency also increases because the plots in a block become more heterogeneous. So we concluded that when block sizes are large and neighbouring plots are highly correlated, generalized least squares for estimation of direct and neighbour effects can be used. The gain in efficiency of NNBD and NNBIBD over regular block design is high under MA(1) models for direct and neighbour effects of treatments.

\section{References:}

[1]. Aastveit, A.H, 1983. On the effect of correlation between plots in randomized block experiments. Biometrical J. 25, 129-153.

[2]. Anderson, A. H., Jensen, E.B., and Schou, G, 1981. Two-way analysis of variance with correlated errors, Int. Statist. Reve. 49, 153167.

[3]. Azais, J.M., Bailey, R. A., and Monod, H, 1993. A catalogue of efficient neighbour designs with border plots, Biometrics, 49, 12521261 .

[4]. Bartlett, M. S, 1978. Nearest neighbour models in the analysis of field experiments (with discussion). J.Roy. Statis. Soc. B 40, 147174.

[5]. Bailey, R. A, 2003. Designs for one-sided neighbour effects, J. Ind. Soc. Agric. Statistics, 56(3), 302-314.

[6]. Besag, J, 1977. Errors-in-variables estimation for Gaussian lattice schemes, J. Roy. Statis. Soc. B 39, 73-78.

[7]. Box, G.E.P, 1954. Some theorems of quadratic forms applied in the study of analysis of variance problems, II. Effects of inequality of variance and of correlation between errors in the two-way classification. Ann. Math. Statist. 25, 484-498.

[8]. Cheng, C. S. and WU, C. F, 1981. Nearly balanced incomplete block designs, Biometrika, 68, 493-500.

[9]. Druilhet, P, 1999. Optimality of neighbour balanced designs, J. Statist. Plan \& Inference, 81, 141-152.

[10]. Gill, P. S. and Shukla, G. K., 1985. Efficiency of nearest neighbour balanced block designs for correlated observations, Biometrika, $72,539-544$

[11]. Kempthorne, O, 1956. The efficiency factor of an incomplete block design, Ann. Math. Statist., 27, 846-849.

[12]. Kunert, J., Martin, R. J., and Pooladsaz, S, 2003. Optimal designs under two related models for interference, Metrika, 57, $137-143$.

[13]. Kunert, J, 1987. Neighbour balanced block designs for correlated errors. Biometrika 74, 4, 717-724.

[14]. Martin, J. P. and Chakravarti, I. M, 1998. Block designs for first and second order neighbour correlations. The Ann. of Statist. 16, 1206-1224.

[15]. Martin, R. J. and Eccleston, J. A, 2004. Variance-balanced designs under interference for dependent observations, J. Statist Plan \& Inference, 119, 207-223.

[16]. Morgan, J.P. and Chakravarti, I.M, 1998. Block Designs for first and second order neithbour correlations. The Ann. Of Statist. 16, 1206-1224.

[17]. Morgan, J.P. and Nizamuddin, 1991. Two dimensional design for correlated errors. The Ann. Of Statist. 19, $2160-2182$.

[18]. Patterson, H.D, 1983. Discussion of paper by G.N. Wilkinson et al. J. Roy. Statis. Soc. B 45, 178-180.

[19]. R. Senthil Kumar \& C. Santharam, Efficiency of Nearest Neighbour Balanced Block Designs using ARMA models, International Journal of Statistics and Systems, ISSN: 0973-2675 Volume 8, Number 1 (2013), pp. 59-71.

[20]. R. Senthil Kumar \& C. Santharam, Efficiency of Nearest Neighbour Balanced Block Designs for correlated observations (ARMA models), International Journal of Statistika and Mathematika, ISSN: 2277-2790 E-ISSN: 2249-8605, Volume 4, Issue 1, 2012, pp 01-05.

[21]. R. Senthil Kumar \& C. Santharam, Efficiency of Neighbour Balanced Block Designs for Correlated Observations, International Journal of Statistika and Mathematika, ISSN: 2277-2790 E-ISSN: 2249-8605, Volume 3, Issue 3, 2012, pp 115-120.

[22]. Santharam.C \& K.N.Ponnuswamy, 1997. On the Efficiency of Nearest Neighbour Balanced Block Designs with Correlated Error Structure, Biometrics, J. 39, 85-98

[23]. Santharam.C \& K.N.Ponnuswamy, 1997. Optimality and Efficiency of Neighbouring Design, Journal of Indian Society of Agricultural Statistics, 50(1), 1997: 1-10

[24]. Tomar, J.S.Jaggi, S., and Varghese, C, 2005. On totally balanced block designs for competition effects, Jour. Applied Statistics, 32 (1), 87-97.

[25]. Tomar, J.S.Jaggi, S, 2007. Efficient neighbour balanced block designs for correlated observations, METRON - International Journal of Statistics, Vol. LXV, n. 2, pp. 229-238.

[26]. Williams, R.M, 1952. Experimental designs for serially correlated observations. Biometrika, 39, $151-167$.

[27]. Wilkinson,G. N., Eckert, S. R., Hancock, T. W., and Mayo, O, 1983. Nearest Neighbour (NN) analysis of field experiments (with discussion). J. Roy. Statist. Soc. B 45,151-2. 